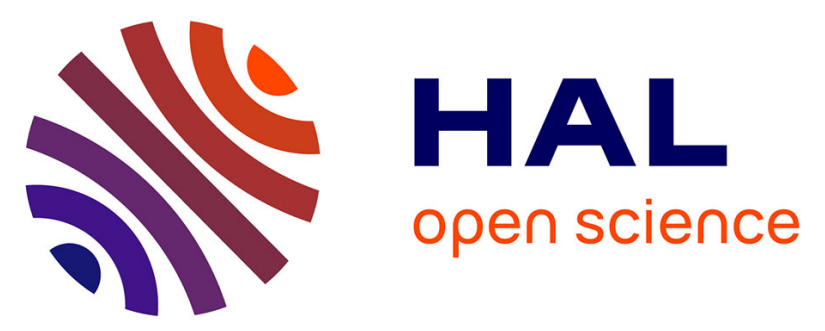

\title{
Microwave Assisted Pure and Mg Doped Tungsten Oxide WO3 Nanoparticles for Superconducting Applications
}

\author{
V. Hariharan, V. Aroulmoji, K. Prabakaran, V. Karthik
}

\section{- To cite this version:}

V. Hariharan, V. Aroulmoji, K. Prabakaran, V. Karthik. Microwave Assisted Pure and Mg Doped Tungsten Oxide WO3 Nanoparticles for Superconducting Applications. International journal of advanced Science and Engineering, 2020, 7 (2), pp.1776-1781. 10.29294/IJASE.7.2.2020.1776-1781 . hal-03093008

\section{HAL Id: hal-03093008 \\ https://hal.science/hal-03093008}

Submitted on 3 Jan 2021

HAL is a multi-disciplinary open access archive for the deposit and dissemination of scientific research documents, whether they are published or not. The documents may come from teaching and research institutions in France or abroad, or from public or private research centers.
L'archive ouverte pluridisciplinaire HAL, est destinée au dépôt et à la diffusion de documents scientifiques de niveau recherche, publiés ou non, émanant des établissements d'enseignement et de recherche français ou étrangers, des laboratoires publics ou privés. 


\title{
Microwave Assisted Pure and Mg Doped Tungsten Oxide W03 Nanoparticles for Superconducting Applications
}

\author{
V. Hariharan ${ }^{1 *}$, V. Aroulmoji ${ }^{2}$, K. Prabakaran ${ }^{1}$, V. Karthik ${ }^{1}$ \\ ${ }^{1}$ PG \& Research Department of Physics, Mahendra Arts and Science College (Autonomous), \\ Kalipatti, Namakkal District, Tamilnadu 637 501, India \\ ${ }^{2}$ Centre for Research \& Development, Mahendra Engineering College, Mahendhirapuri, \\ Mallasamudram 637503, Namakkal District, Tamil Nadu, India
}

ABSTRACT: The aim of the present work focuses the role of "Mg" in W03.H2O nanopowders, doped with Magnesium using a facile microwave irradiation process and the annealing process was carried out at $600^{\circ} \mathrm{C}$ in air for $6 \mathrm{~h}$ in order to remove the impurities and enhance the crystallinity of the end products. The samples were further characterized by powder X-ray diffraction, field emission scanning electron microscopy, UV-VIS diffusion reflectance spectroscopy, and the relevant magnetic behaviors of the products were carried out using vibrational sample magnetometer to find out the suitability of the material for superconducting applications. Powder X-ray diffraction confirmed that the samples both undoped and doped WO3.H2O crystallized with orthorhombic phase respectively. On the other hand, annealing effect on the samples formed with orthorhombic phase. It clearly shows the doping on crystalline nature of the samples was also monitored using power X-ray analysis. Observations from FE-SEM micrographs suggested that the samples having influence with dopants in growth rate along with morphology of the prepared nanoparticles. UV-VIS-DRS spectroscopy was an important tool used to find the optical band gap of these samples and the results revealed the contribution of the dopant materials in the the optical band gap values using KM model and the corresponding blue shift was observed with $360 \mathrm{~nm}$ wavelength. The interesting results from hysteresis loops from vibrational sample magnetometer on the annealed samples showed that the doped samples try to explore well into the superconducting or diamagnetic state reveal the suitability of the prepared materials for superconducting applications.

KEYWORDS: Tungsten oxide, Microwave irradiation, Cobalt doping, Nanoparticles, TEM, VSM

\section{INTRODUCTION}

Nanosized materials have attracted great attention as a result of exhibiting unique surface to volume ratio. In particular, high surface area materials have been of great interest in a wide range of applications such as catalysis, chemical and biosensors, fuel cell electrodes and so forth [1]. As a well known inorganic oxide, W03 is a promising candidate for many applications such as electrochromic [2], photocatalytic [3], photoluminescent [4] and as a gas sensor device [5] due to their existence of various structural polymorphs and easily tunable oxygen content of the end product by varying the growth atmosphere. In fact, the optical and electrical properties of this compound strongly depend on size and morphology of the corresponding end product. Accordingly, the recent scenarios for many practical applications are mainly based on morphology and size distribution of the nanoparticles. This can be done by varying the synthesis procedure and growth atmosphere which influence the morphology and size distribution of the nanoparticles. On the other hand, dopants have offered relatively better morphology and high surface to volume ratio of the nanoscale materials. To date, following methods have been adopted to synthesis pure and doped W03 in the form of powders; vapor deposition [6], hydrothermal route [7], sol-gel [8], acidification method [9], electrospinning method [10], electro deposition method [11] etc. However, the above mentioned techniques are more time consuming and cost effective. Great efforts are being taken to explore new synthesis methods to control the morphology and size distributions to satisfy certain applications. Recently, most of the works focused on the preparation of doped nanocrystalline WO3 following their successful applications. Zhu et al. have prepared $\mathrm{Cu}-$ doped WO3 materials with photonic structures using combined sol-gel templating and calcinations method for the detection of various volatile organic gases. They 
suggested that the photonic crystal of Cu-doped WO3 replica sensor has a much higher response as well as selectivity when compared to that of pure one due to their different morphologies [12]. Cheng et al fabricated Zn-doped WO3 thin films and found the enhanced behavior of Zn doped WO3 with a suitable amount in the case of photocurrent and photo-activity [13]. Zamani et al. have synthesized Cr-doped mesoporous W03 nanomaterial by chemical route for the detection of amines and tetra methyl amines. Finally they summarized the product showed low sensitivity to NH3 and TMA was detected more efficiently [14]. Xia et al. have prepared pure and Audoped W03 nanopowders by a colloidal chemical method. They concluded that proper Au loading on W03 is suitable for the detection of NO2 at relatively low temperature [15]. Kalidindi et al. fabricated Tidoped W03 films on Si(100) wafers using sputtering technique and found that the electrical conductivity of Ti-doped samples at room temperature is more than that of pure sample [16].

With this basis, bear in mind the role of dopants in nanocrystalline W03.H2O and W03, we have been able to synthesis cobalt doped W03.H2O nanopowders with different $\mathrm{W} /$ Co (0.02 and 0.05 ) ratios using simple, efficient straight forward microwave irradiation method. The procedure reported here, to the best of our knowledge, is the first demonstration in the synthesis of cobalt doped W03.H2O nanopowders. In the microwave irradiation method, time required for the synthesis was around 10 min only and the reaction process was also very simple [17].

\section{EXPERIMENTAL DETAILS}

Pure and doped hydrated tungsten oxide (W03.H20) nanoparticles were prepared by microwave irradiation method at room temperature and without employing hydrothermal method during synthesis process [18]. Analytical grade of $4.98 \mathrm{~g}$ (around one molar ratio) of tungstic acid (H2W04) was dissolved in $20 \mathrm{~mL}$ of sodium hydroxide $(\mathrm{NaOH})$. The obtained solution was yellow in color and it was stirred for $20 \mathrm{~min}$ in ambient condition. This may be due to proton exchange protocol process [19]. Subsequently, 2 wt.\% and 5 wt.\% of cobalt Manganese Chloride $(\mathrm{MgCl} 2)$ was mixed along tungstic acid which was added into $20 \mathrm{~mL}$ of deionized water. The final solution was slowly mixed together and stirred again for $20 \mathrm{~min}$. The $\mathrm{pH}$ of the solution was found to be neutral (7.0) due to salty nature of the mixed solution and it was adjusted to 1 with the addition of $\mathrm{HCl}$ because it can act as a precipitating agent and also medium for the product to have desired morphology [20]. In addition with that above solution $5 \mathrm{ml}$ double distilled water (i.e. 50 vol. $\%$ of precursor solution) was added with the above solution in order to respond to microwave quickly. The final solution of both pure and doped samples were transferred into a microwave oven $(2.45 \mathrm{GHz}$ and maximum power of $900 \mathrm{~W}$ ) in ambient condition and kept into $600 \mathrm{~W}$ for $10 \mathrm{~min}$. The resultant yellow precipitate was annealed in a tubular furnace at $600 \mathrm{C}$ for $6 \mathrm{~h}$ in air to remove the sub products and to improve the crystallanity.

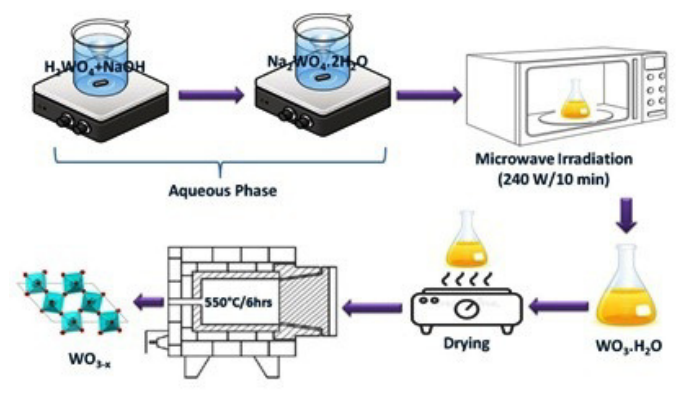

Fig. 1 Schematic illustration of synthesis of doped W03.H2O in microwave atmosphere

\section{Characterizations Powder XRD Analysis}

Fig. 2 reveals the obtained powder XRD pattern for doped hydrated tungsten oxide and Fig. 3 shows the pattern for respective annealed samples. The powder XRD patterns of pure W03.H2O and annealed WO3- $\delta$ have been analyzed in the previous works in details as mentioned as reference. The Powder XRD results confirmed the orthorhombic phase formation of WO3.H2O with improved crystalline nature in agreement with JCPDS reference no: 43-0679. Also it is to be noted that a small shift in smaller Bragg angle side for doped (0.02) sample indicating the incorporation of dopant ion in W03.H2O crystalline lattice due to significant atomic radii of dopant ion. On the other hand, the re attainment of crystallite site for manganese (5 Wt.\%) was nearly to the Bragg's value of pure sample along (020) plane clearly resembled the incorporation of $\mathrm{Mg}$ ion in WO 2- (020) tetrahedron structure not in water molecule site (111) which is another confirmation for the attainment of original parent phase nature.

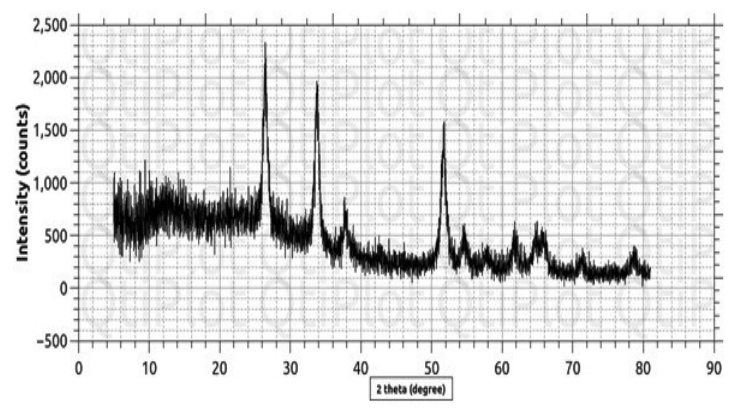

Fig. 2: Representative Powder XRD pattern of doped W03.H20

Hariharan et al., 


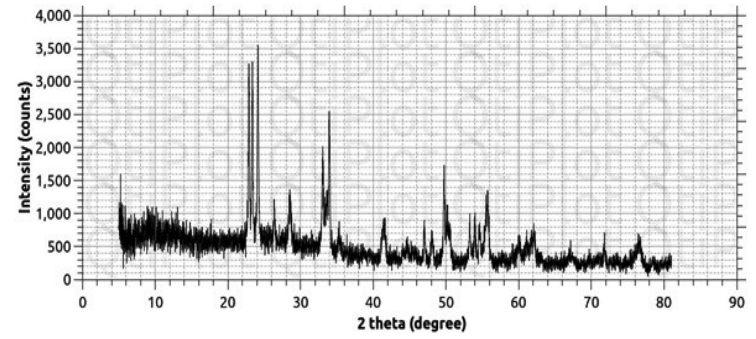

Fig. 3: Representative Powder XRD pattern of annealed (doped) W03

\section{Microscope Analysis (FE-SEM)}

The microscope analysis was carried out for the resultant as prepared and annealed nanoparticles of pure and doped tungsten oxides on a aluminum substrates are shown in Fig. 4. This FE - SEM resembles the formation of sphere like morphology agglomeration having in the order of 1-2.5 $\mu \mathrm{m}$ in longer axis and 1-2 $\mu \mathrm{m}$ in shorter axis. Also as additional information the close examination revealed that plates lying parallel to the surface of the substrate and normal to surface are rare that clearly indicating the growth along $b$ axis corresponding to (020) plane which is in agreement with the Powder XRD analysis. Above all this phenomenon arising in edge effects were also arised due to charge transfer between ' $W$ ' and ' $O$ ' ions present in the plates that has been marked in the Fig. 4.

Fig. 5 shows the annealed pure and doped W03nanoparticles. It is also having with agglomerate in nature with poly disperse nature with sphere like morphology of the order of $4-5 \mu \mathrm{m}$ in lateral axis and $2-3 \mu \mathrm{m}$ in longitudinal axis. This may be due to the fact that the agglomeration of fast and rapid collision between the particles during the recrystallization process because of annealing effect. The attainment of highly uniform with high crystalline nature than the pure sample illustrated the role of dopant in modifying the morphology of the end products especially annealed samples. Also, oxygen content also plays an important role in fixing the condition for morphology, because the percentage of oxygen in Magnesium doped sample is more than that of pure sample.

\section{UV-VIS Diffusion Reflectance Analysis $(U V-V I S-D R S)$}

The UV VIS diffuse reflectance spectroscopy was carried out to know the optical properties of the pure and doped as prepared and annealed samples are shown in Fig. 6. There are strong and wide reflectance peaks between the region 500-650 $\mathrm{nm}$ which indicated the prepared nanoparticles can display optical conductivity in the visible region. The maximum reflectance intensities have obvious blue shifts (as prepared samples) and red shifts (annealed samples) within the spectral region 500-650 $\mathrm{nm}$.

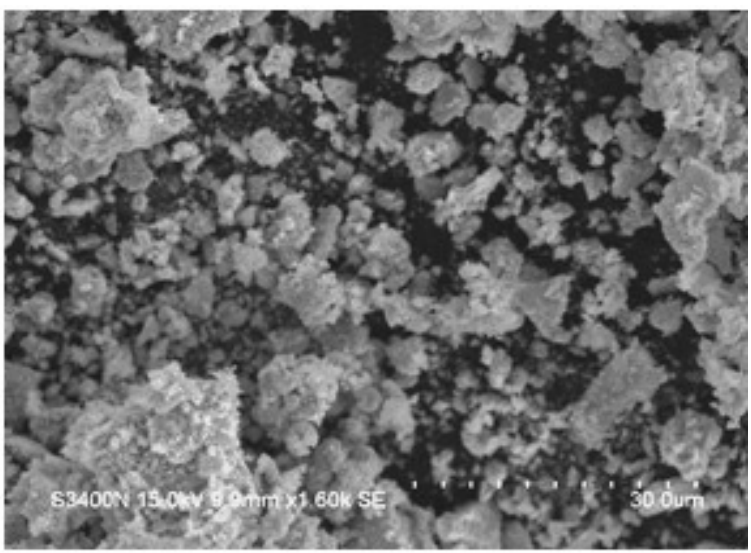

Fig. 4: FE-SEM images of WO3.H2O doped

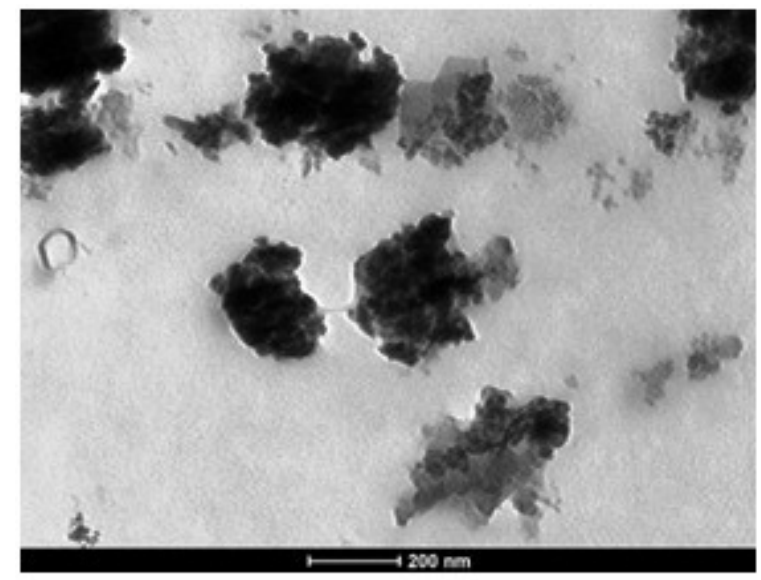

Fig. 5: FE - SEM images of WO3 doped

The band gap energies for all the samples may be determined by using Kubelka-Munk (K-M model and its corresponding relation is given below.

$$
\frac{K}{S}=\frac{\left(1-R_{\infty}\right)^{2}}{2 R_{\infty}}=F\left(R_{\infty}\right)
$$

$F(R)$ is the so called remission or Kubelka - Munk function, where

$$
R_{\infty}=-\begin{gathered}
R_{\text {sample }} \\
R_{\text {std }}
\end{gathered}
$$

A graph is plotted between $[\mathrm{F}(\mathrm{R} \infty) \mathrm{hv}]^{2} \mathrm{Vs} \mathrm{hv}$ and the intercept value is the band gap energy Eg of the individual sample [21]. The band gap energies as a function of Magnesium concentration were found to be

\section{Hariharan et al.,}


3.27 and $3.33 \mathrm{eV}$ respectively. On the contrary, in the case of annealed samples the band gap energies in the identical doping conditions to be 2.87 and $3.44 \mathrm{eV}$ respectively.

The reason for the increase in Eg (as prepared) may be explained using Burstein-Moss (BM) [22] effect in which the lowest states in the conduction band are blocked and the allowed transitions can take place only to energies above Fermi level to unblocked valance level (between $\mathrm{W}^{6+}$ to $\mathrm{Mg}^{2+}$ ) due to the introduction of $\mathrm{Mg}^{2+}$ ion in the intermediate energy state. On the other hand, in the case of annealed samples, the increase in band gap energies as a function of dopant concentration is due to the fact that exchange interaction between the band electrons of $d x 2-y 2$ and dxy orbital of tungsten to the dx2-y2 and dz2 orbital of $\mathrm{Mg}^{2+}$ ion. It gives rise to the positive correction in the valance band and leads to narrowing the band gap (Fig. 6).

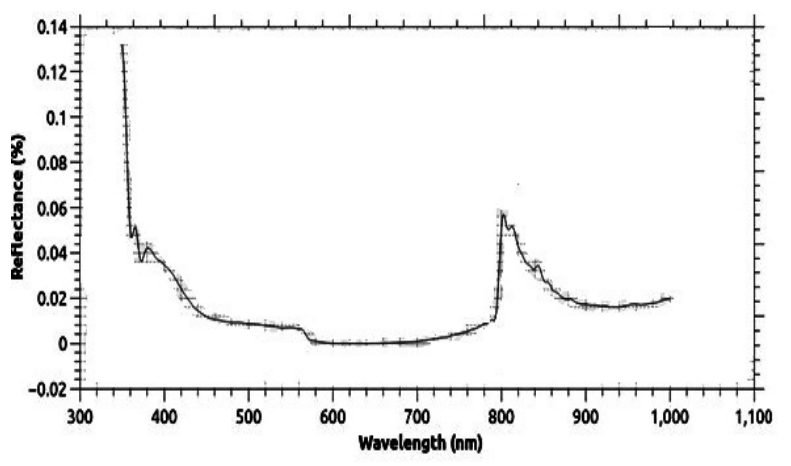

Doped W03.H20

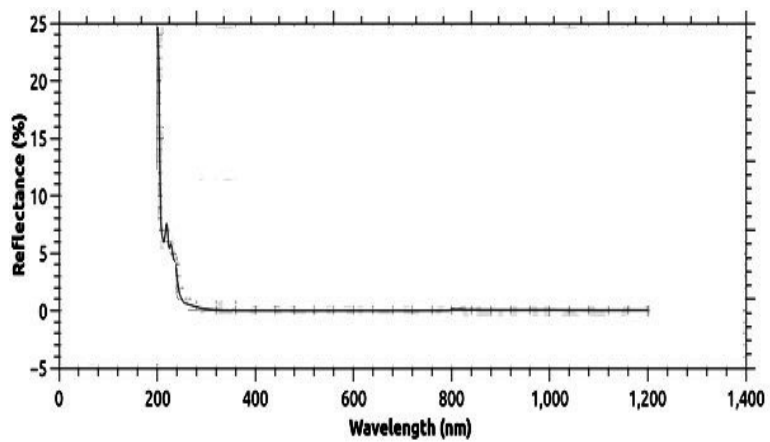

Fig. 6: UV - VIS - DRS plots of doped W03.H20 and WO3

\section{Magnetic behavior (VSM) Analysis}

Fig. 7 presents the hysteresis loops measured at room temperature of pure and Magnesium doped respective tungsten oxide (WO3) nanoparticles. The measurements were performed on annealed samples since it has high crystalline nature with significant oxygen vacancies. It is to be understood that stoichiometric tungsten oxide may be exhibited dia magnetic behavior at room temperature and suits for superconducting applications [23]. Interestingly, The present prepared annealed both pure and doped exhibits the co existence of both dia and ferromagnetic behavior [24] of the samples and may be attributed to the fact that the in homogeneity in holes density due to the lack of oxygen (W17047) which gives rise to the unpaired electrons in holes rich region enhanced the ferromagnetic behavior in addition with the dia magnetic nature. Subsequently, it can seen from the Magnesium doped samples which are having light excess of oxygen than pure sample could enhance the diamagnetic behavior at negative magnetic field due to its stoichiometric oxygen content of the resultant annealed samples. These unexpected results suggest that the doped samples try to persist well into the superconducting or diamagnetic state, namely the coexistence of dia and ferromagnetism in the hole rich clusters. The enhancement of dia magnetic behavior in doped samples with respect to the relative suppression of ferro magnetic behavior indicate the role of dopant in fixing the oxygen content and magnetic behavior of the end products especially annealed samples.

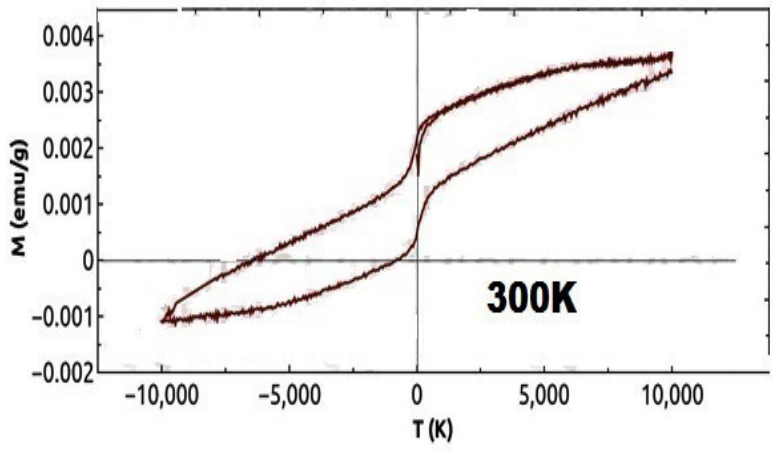

Fig. 7: Room temperature hysteresis loops of W03- D Doped

\section{CONCLUSIONS}

The present work focuses the successful completion of pure and doped W03.H2O by efficient microwave irradiation method in ambient atmosphere. The successive crystalline phase of all the end products found that (Pure and Doped) with orthorhombic in phase. In the case of annealed samples pure and doped samples (5wt.\%) were found to be became orthorhombic structure from Powder XRD analysis. Hence, these unexpected results suggested the role of dopants in fixing the oxygen content of the end product. UV-VIS-DRS studies will be a evidence in order to know that the as prepared samples having low optical conductivity than that of the annealed samples. This

\section{Hariharan et al.,}


observation recommends the required optical conductivity can be attained by doping elements itself instead of annealing process. The interesting results from hysteresis loops suggested that the doped samples try to persist well into the superconducting or diamagnetic state than pure sample suggested that $\mathrm{Mg}$ doped samples in association with WO3 crystalline phase will be a promising candidate for superconducting applications.

\section{REFERENCES}

[1] Tahir. M. B., Nabi. G., Rafique. M. Khalid. N.R, 2017. Nanostructured based W03 photo catalysts: recent development, activity enhancement, perspectives and applications for wastewater treatment. Int. J. Environ. Sci. Technol. 14, 2519-2542.

[2] Saasa. V, Lemmer. Y, Malwela. T, Akande. A, Beukes. M, Mwakikunga. B, 2020. Effect of varying ethanol and water compositions on the acetone sensing properties of W03 for application in diabetes mellitus monitoring. Mater. Res. Express, 7, 035905.

[3] Hameed. A, Gondal. M. A, Yamani. Z. H, 2004. Effect of transition metal doping on photocatalytic activity of W03 for water splitting under laser illumination: role of $3 \mathrm{~d}$-orbitals. Catal. Commun. 5, 715-719.

[4] Baig. U, Gondal M. A. , Suriya Rehman, Sultan Akhtar,2020. Facile synthesis, characterization of nano - tungsten trioxide decorated with silver nano particles and their antibacterial activity against water-borne gram - negative pathogens. Appl Nanosci, 10, 851-860.

[5] Duan. G, Chen. L, Jing. Z, Luna. P. D., Wen. L, Zhang. L, Zhao. L, Xu. J, Li. Z, Yang. Z, Zhou. R, 2019. Robust Antibacterial Activity of Tungsten Oxide (W03-X) Nanodots. Chem. Res. Toxicol, 32(7), 1357-1366.

[6] Matharu. R. K., Ciric. L, Ren. G, Edirisinghe. M, 2020. Comparative Study of the Antimicrobial Effects of Tungsten Nanoparticles and Tungsten Nanocomposite Fibres on Hospital Acquired Bacterial and Viral Pathogens. Nanomaterials 10, 1017,1 - 16.

[7] Jeevitha. G, Abhinayaa. R, Mangalaraj. D, Ponpandian. N, 2018. Tungsten oxide-graphene oxide (WO3-GO) nanocomposite as an efficient photocatalyst, antibacterial and anticancer agent. J Phys Chem Solids,116, 137-147.

[8] Han. B, Popov. A. L, Shekunova. T. O, Kozlov. D. A, Ivanova. 0. S, Rumyantsev. A. A, Shcherbakov. A. B, Popova. N. R, Baranchikov. A. E, Ivanov. V. K, 2019. Highly Crystalline WO3 Nanoparticles Are Nontoxic to Stem Cells and Cancer Cells. J. of Nanomaterials, ID 5384132.
[9] Ahamed. M, Hisham A. Alhadlaq, Majeed Khan. M. A, Karuppiah. P, Naif A. Al-Dhabi, 2014. Synthesis, Characterization, and Antimicrobial Activity of Copper Oxide Nanoparticles. J. of Nanomaterials, 2014

[10] Ghasempoura. F, Azimiradb. R, Aminic. A, Akhavanb. O, 2015. Visible light photoinactivation of bacteria by tungsten oxidenanostructures formed on a tungsten foil. Appl. Surf. Sci. 338, 55 - 60.

[11] Bankier. C, Matharu. R. K, Cheong. Y. K, Ren. G. G, Cloutman-Green. E, Ciric. L,2019. Synergistic Antibacterial Effects of Metallic Nanoparticle Combinations. Sci. Rep. 9.

[12] Syed. M. A, Manzoor. U, Shah. I, Habib Ali Bukhari. S, 2010. Antibacterial effects of Tungsten nanoparticles on the Escherichia coli strains isolated from catheterized urinary tract infection (UTI) cases and Staphylococcus aureus. New Microbiol, 33, 329-335.

[13] Ismail. R. A, Sulaiman. G. M, Abdulrahman. S. A, Marzoog. R. T, 2015. Antibacterial activity of magnetic iron oxide nanoparticles synthesized by laser ablation in liquid. Mater. Sci. Eng. C. 53, 286-297.

[14] Turkez. H, Sonmez. E, Turkez. O, Mokhtar. Y. I, Stefano. A. D, Turgut. G, 2014. The Risk Evaluation of Tungsten Oxide Nanoparticles in Cultured Rat Liver Cells for Its Safe Applications in Nanotechnology. Braz Arch Biol, 57, 532-541.

[15] Popov. A. L, Savintseva. I. V, Popova. N. R, Shekunova. T. O, Ivanova. O. Shcherbakov. A. B, Kozlov. D. A, Ivanov. V. K. 2019. PVP stabilized tungsten oxide nanoparticles (W03) nanoparticles cause hemolysis of human erythrocytes in a dose dependent manner. Nanosystems: Physics, Chemistry, Mathematics, 10, 199-205.

[16] Akbaba. G. B, Turkez. H, Sonmez. E, Akbaba. U, Aydın. E, Tatar. A, Turgut. G, Cerig. S, 2016. In vitro genotoxicity evaluation of tungsten (VI) oxide nanopowder using human lymphocytes. Biomed. Res. 27, 125-130.

[17] Hariharan, V., Aroulmoji, V., Sekar, C., Bhuvaneshwari, R. S., Pathmavathi, V., Kavina, K., Anbalagan. A. 2016. Polyethylene Glycol (PEG) Assisted Tungsten Oxide (W03) Nanoparticles by Microwave Irradiation Method, Int. J. Adv. Sci. Eng 3 (2), 278-255

[18] Chiu. Y. H, Mark. T. F, Chang, Chen. Y. C, Sone. M, Hsu. Y. J.2019. Mechanistic Insights into Photo degradation of Organic Dyes Using Hetero structure Photocatalysts, Catalysts, 9,430,

[19] Palanisamy. G, Bhuvaneswari. K, Pazhanivel. T, Bharathi. G, 2020. Enriched, photocatalytic activity of Rhodamine $\mathrm{B}$ dye from aqueous solution using hollow sphere tungsten trioxide nanoparticles. Optik, 204,

Hariharan et al., 
[20] Arshad. M, Ehtisham-ul-Haque. S, Bilal. M, Ahmad. N, Ahmad. A, Abbas. M, Nisar. J, Khan. M. I, Nazir. A, Ghaffar. A, Iqbal. M, 2020. Synthesis and characterization of Zn doped W03 nanoparticles: Photocatalytic, antifungal and antibacterial activities evaluation. Mater. Res. Express, 7(1).

[21] Hariharan.V, Parthibavarman. M, Sekar. C, 2011. Synthesis of tungsten oxide (W18049) nanosheets utilizing EDTA salt by microwave irradiation method. J. of Alloy. And Comps. 509, 4788-4792, 2011.

[22] Hariharan, V, Radhakrishnan, S, Parthibavarman, M, Dhilipkumar. R, Sekar. C. 2011. Synthesis of polyethylene glycol (PEG) assisted tungsten oxide (WO3) nanoparticles for l-dopa biosensing applications. Talanta, 85, 2166-2174.
[23] Sarmiento Arellano. J. S, Vega. A. K, RosendoAndrés. E, Díaz-Becerril. T, Romano- Trujillo. R, Oliva. A. I, De la Cruz. W, Lugo. J. M, MoralesRuíz. C, Galeazzi-Isasmendi. R, García-Salgado. G, Nieto. F. G, 2016. Influence of $\mathrm{HCl}$ on the NPsCdSe synthesis prepared by the colloidal method. J Appl Res Technol. 14, 225-231.

[24] Saleh. T. A, Principles and Advantages of Microwave-Assisted Methods for the Synthesis of Nanomaterials for Water Purification. IGI Global Publisher of Timely Knowledge. (2017),

All () 2020 are reserved by International Journal of Advanced Science and Engineering. This Journal is licensed under a Creative Commons Attribution-Non Commercial-ShareAlike 3.0 Unported License.

Hariharan et al., 